\title{
Application of Image Compression Based on Discrete Cosine Transform
}

\author{
Hongmei Zhang \\ College of Computer Science and Technology \\ Inner Mongolia University for Nationalities \\ TongLiao, China \\ e-mail: Tongyu_007@163.com
}

\author{
Zhigao Zhang \\ College of Math \\ Inner Mongolia University for Nationalities \\ TongLiao, China \\ e-mail: zzgtongxin@163.com
}

\author{
Zhili Pei * \\ College of Computer Science and Technology \\ Inner Mongolia University for Nationalities \\ TongLiao, China \\ e-mail: zhilipei@sina.com \\ *corresponding author
}

\begin{abstract}
Image compression encoding is one of the key techniques in modern multimedia and communication field. DCT is one of the most widely used in image compression processing. It uses the symmetry of the Fourier transform and performs a folding operation on the boundary of the image. It transforms the image into even function form. DCT has been widely used in the areas such as image compression and other fields due to its good energy compaction property and fast computation. In recent years, DCT is one of the basic processing modules in a series of international standard of video coding. Especially, the DCT transform is used in the JPEG and the MPEG. This paper briefly introduces the concept and theory of DCT. The paper introduces the core of DCT coding, and uses the MATLAB language to achieve the DCT-based image compression.
\end{abstract}

Keywords-Data Compression; Image Compression; Discrete Cosine Transform; Joint Photography Experts Group Compression Encoding; Coding

\section{INTRODUCTION}

With the rapid development of communication and information technology, digital information grows explosively [1]. In this process, data compression technology plays an important role in people's life, work and scientific research. As an important branch of data compression field, discrete cosine transform (DCT) is considered as a good method in theory ${ }^{[2]}$. This paper is based on DCT to study a small branch of data compression in the field of image compression. Image compression technology is one of the key technologies in the field of modern multimedia and communication technology. Discrete cosine transform (DCT) is widely used in image compression due to its better energy compression and fast algorithm. In recent years, based on DCT transform analysis, processing operations research is very active, especially the static image compression standard JPEG and dynamic image compression standard MPEG using DCT transform, more to promote the development of this field ${ }^{[3]}$. Therefore based on the DCT transform of the image compression technology is also rapidly developing. This paper describes the core of the DCT encoding, and the use of MATLAB language to achieve the image compression based on DCT.

\section{ALGORITHM BASED ON DCT IMAGE COMPRESSION}

\section{A. DCT Profile}

Discrete cosine transform (Cosine Transform DCTDiscrete), by Ahmed and Rao in 1974, has a history of 30 years ${ }^{[4]}$. Here, DCT encoding has developed into a BMP, H.26x, MPEG and other image / image of the core of encoding standard. DCT is a kind of spatial transformation, the biggest characteristic of DCT transform is that general images are able to block the energy to focus on a few lowfrequency DCT coefficients, so that you may only encode and transmit a few coefficients without seriously affecting the quality of the image. DCT can not directly produce a compression effect on the image, but the image of the energy has a very good concentration effect, which is based on the compression. For example, the image of one frame content is represented by different luminance and color value pixels, and the distribution of these pixels is changed according to the image content. However, by the discrete cosine transform (DCT), the distribution of pixels is regular. The amount of the low frequency component is distributed in the upper left corner, and the higher the frequency component is to the lower right corner ${ }^{[5]}$. Then according to the human visual characteristics, remove some details (high frequency components) which do not affect the basic content of the image, so as to achieve the purpose of the compression rate.

\section{B. Some Concepts of Discrete Cosine Transform}

- One dimensional discrete cosine transform Positive transformation:

$$
F(k)=2 \sum_{n=0} f(n) \cos \frac{\pi(2 n+1) k}{2 N}
$$


Where $\quad n, k=0,1,2 \cdots N-1$

Inverse transformation:

$$
\begin{aligned}
& f(n)=\frac{1}{N} \sum_{n=0}^{N-1} F(k) \cos \frac{\pi(2 n+1) k}{2 N} \\
& \text { Where } \quad n, k=0,1,2 \cdots N-1
\end{aligned}
$$

- Two dimensional discrete cosines transform Positive transformation:

$$
F(u, v)=c(u) c(v) \sum_{x=0}^{M-1} \sum_{y=0}^{N-1} f(x, y) \cos \frac{\pi(2 x+1) u}{2 M} \cos \frac{\pi(2 y+1) v}{2 N}
$$

Where: $u=0,1, \cdots M-1 ; \quad v=0,1, \cdots N-1$

$$
\begin{aligned}
& c(u)=\left\{\begin{array}{lc}
\sqrt{1 / M} & u=0 \\
\sqrt{2 / M} & u=1,2, \ldots M-1
\end{array}\right. \\
& c(\mathrm{v})= \begin{cases}\sqrt{1 / N} & u=0 \\
\sqrt{2 / N} & u=1,2, \ldots N-1\end{cases}
\end{aligned}
$$

Inverse transformation:

$f(x, y)=\sum_{x=0}^{M-1} \sum_{y=0}^{N-1} c(u) c(v) f(u, v) \cos \frac{\pi(2 x+1) u}{2 M} \cos \frac{\pi(2 y+1) v}{2 N}$

- Simplification of two dimensional discrete cosine transform

In the two-dimensional discrete cosine transform, $\mathrm{x}, \mathrm{y}$ is the spatial sampling value, and it is usually used in the digital image pixel matrix, that is, in the case of $\mathrm{M}=\mathrm{N}$, the two-dimensional discrete cosine transform can be simplified to:

$f(u, v)=\frac{2}{N} \sum_{x=0}^{M-1 N-1} \sum_{y=0} c(u) c(v) f(x, y) \cos \frac{\pi(2 x+1) u}{2 M} \cos \frac{\pi(2 y+1) v}{2 N}$

Where

$$
c(u) c(v)=\left\{\begin{array}{cc}
1 / \sqrt{2} & u=0 \quad \text { or } \quad v=0 \\
1 & u, v=1,2, \cdots N-1
\end{array}\right.
$$

In MATLAB, $c(u) c(v) \cos \frac{\pi(2 x+1) u}{2 M} \cos \frac{\pi(2 y+1) v}{2 N}$ is called the basic function of the discrete cosine transform, so that the coefficients of the DCT can be regarded as the weighted of each basis function.

\section{IMAGE COMPRESSION ENCODING OF DCT}

\section{A. DCT Encoding}

Any continuous real symmetric functions of the Fourier transform only contains the cosine term, so the cosine transform and Fourier transform as well as a clear physical meaning. DCT is the first to divide the whole image into $\mathrm{N} * \mathrm{~N}$ pixel block, and then the $\mathrm{N} * \mathrm{~N}$ pixel block DCT transform ${ }^{[6]}$. Because most of the image of the high frequency component is smaller, the corresponding to the coefficients of high frequency components of an image is always zero, and eye of the high frequency component distortion less sensitive, so more coarse quantization is available, so the transport transform coefficients of digital rate to considerably less than the transmission of image pixels of digital rate ${ }^{[7]}$. After the arrival of the receiving end of the inverse discrete cosine transform to the sample value, although there will be some distortion, but the human eye is acceptable.

After a DCT operation of the original input image, the output matrix of the results has a characteristic: When the element is getting further and further away from the DCT coefficient, its module is getting smaller and smaller. This means that, after the useful information of the image is concentrated in the upper left corner of the matrix, and the right lower part of the matrix is almost no useful information. In brief, the whole image is divided into $\mathrm{N} *$ $\mathrm{N}$ pixel blocks, then the $\mathrm{N} * \mathrm{~N}$ pixel blocks are transformed by DCT one by one. Due to the high frequency component of most images less and the coefficients of the corresponding high frequency components of an image are always zero. Moreover, the human eye is not sensitive to the distortion of high frequency components, so the high frequency components of the image can be quantified roughly ${ }^{[8]}$. The digital rate of the transmission coefficient is far less than the rate of transmitted image pixels. After the transfer results are received, the sample value is returned by the inverse discrete cosine transform, although there will be some distortion, but the human eye is acceptable ${ }^{[9]}$. N represents the number of pixels. Under normal circumstances, $n=8$. After DCT data processing, the two - dimensional data block, which size is $8 * 8$, is transformed into $8 * 8$ transform coefficients. These coefficients have a clear physical meaning: $U$ represents the horizontal pixel number, and $\mathrm{V}$ represents the vertical pixel number. Such as $\mathrm{U}=0, \mathrm{~V}=0, \mathrm{~F}(0,0)$ is the average of the original 64 samples, equivalent to DC component. With the increase of $\mathrm{U}$ and $\mathrm{V}$, the corresponding coefficients represent respectively the size of gradually increasing frequency component of horizontal space and vertical space.

\section{B. Coefficients Quantization}

The so-called quantization is to discrete the instantaneous value of the sample, which uses a set of agreed electrical levels, and the instantaneous sampling value is represented by the nearest level value. The whole amplitude is divided into several quantization levels, and to class the sample values at the same level together and give a quantization value. The more quantified levels we divide, the lower quantified error we get, and the better sound quality we get ${ }^{[10]}$

In process of image quantization, firstly, the image is transformed from the time domain to the frequency domain, for different frequency corresponds to different coefficient, different coefficients according to the certain level of quantization will be rounded and become small. The new frequency domain graph obtained is the frequency domain of the compressed image. The role of quantization is to ensure the quality of the image in a certain extent, to lose those of the visual impact of the information, in order to obtain a higher compression ratio. 
However, it is also the main reason for the decrease of image quality. In this paper, we use the quantization matrix to realize the quantification. The quantization formula is as follows:

$$
\begin{aligned}
& Q(u, v)=\text { IntegerRound }(F(u, v) / S(u, v)) \\
& Q(u, v) \text { is the amplitude of quantized coefficients, }
\end{aligned}
$$

$S(u, v)$ is the quantization step size, which is the element of the quantization table and different from the position of the DCT coefficient and the size of color components. The size of the quantization table is $8 * 8$, corresponding to 64 DCT coefficients.

\section{ACHIEVE DCT IMAGE COMPRESSION BASE ON MATLAB}

MATLAB is a kind of high performance programming software for engineering calculation. Its function is powerful, and its application is wide. Image processing is one of the most important applications. In the process of image transformation using discrete cosine transform base on MATLAB, first, the input image is decomposed into 8 * 8 blocks, then two - dimensional discrete transform is performed for each block, finally the DCT coefficients after transformation are encoded and transmitted. Instead, the decoding is performed on each block by twodimensional DCT inverse transformation. Then, after the inverse transformation, the block is combined into an image.

For most images, most of the value of the DCT coefficient is close to 0 , if abandon these values close to 0 , in the reconstructed image, the image quality is not significantly decreased. Therefore, the use of DCT for image processing can save a lot of storage space. Compression should be used in the most reasonable approximation of the original image using the least coefficient. According to the above method, an image is divided into $8 * 8$ block for compression. The implementation method is as follows

$$
\begin{aligned}
& \text { function } \mathrm{Y}=\operatorname{dct} 21(\mathrm{X}) \\
& {[\mathrm{m}, \mathrm{n}]=\operatorname{size}(\mathrm{X}) ;} \\
& \mathrm{AM}=\operatorname{zeros}(\mathrm{m}, \mathrm{m}) ; \\
& \mathrm{n}=8 \text {, } \operatorname{AM}(\mathrm{AN}) \text { is the } \\
& \mathrm{mmonly} \text { used in JPEG. } \\
& \mathrm{AN}=\text { zeros }(\mathrm{n}, \mathrm{n}) ; \\
& \text { for } \mathrm{i}=0: \mathrm{m}-1 \\
& \text { for } \mathrm{j}=0: \mathrm{m}-1 \\
& \text { if } \mathrm{i}==0
\end{aligned}
$$

$\mathrm{AM}=\operatorname{zeros}(\mathrm{m}, \mathrm{m}) ; \quad \%$ The coefficient of DCT, When $\mathrm{m}=\mathrm{n}=8, \mathrm{AM}(\mathrm{AN})$ is the $8 * 8$ transformation matrix commonly used in JPEG.

$\operatorname{AM}(\mathrm{i}+1, \mathrm{j}+1)=\operatorname{sqrt}(1 / \mathrm{m}) * \cos (((2 * \mathrm{j}+1) * \mathrm{i} * \mathrm{pi}) /(2 * \mathrm{~m}))$;

else

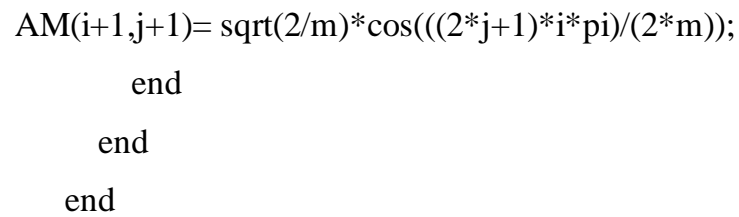

for $\mathrm{i}=0: \mathrm{n}-1$

$$
\begin{gathered}
\text { for } j=0: n-1 \\
\text { if } i==0
\end{gathered}
$$

$\mathrm{AN}(\mathrm{i}+1, \mathrm{j}+1)=\operatorname{sqrt}(1 / \mathrm{n}) * \cos (((2 * \mathrm{j}+1) * \mathrm{i} * \mathrm{pi}) /(2 * \mathrm{n}))$;

else

$\operatorname{AN}(i+1, j+1)=\operatorname{sqrt}(2 / n) * \cos (((2 * j+1) * i * p i) /(2 * n))$;

end

end

end

$\mathrm{X}=\operatorname{double}(\mathrm{X}) ; \%$ The type of Matrix data transformed is double.

$$
\mathrm{Y}=\mathrm{AM} * \mathrm{X}^{*} \mathrm{AN}
$$

The implementation effect of the code is shown in Figure 1.

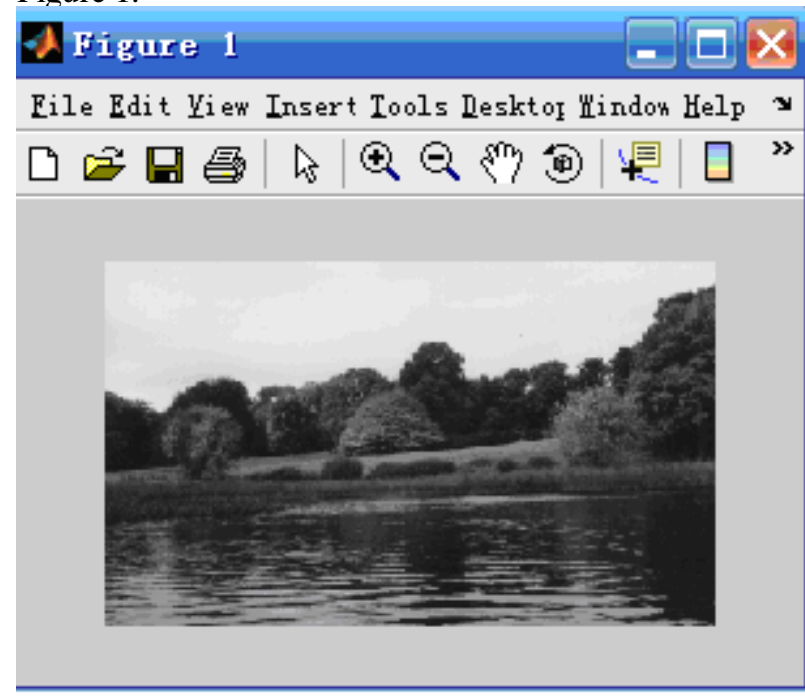

Original Image

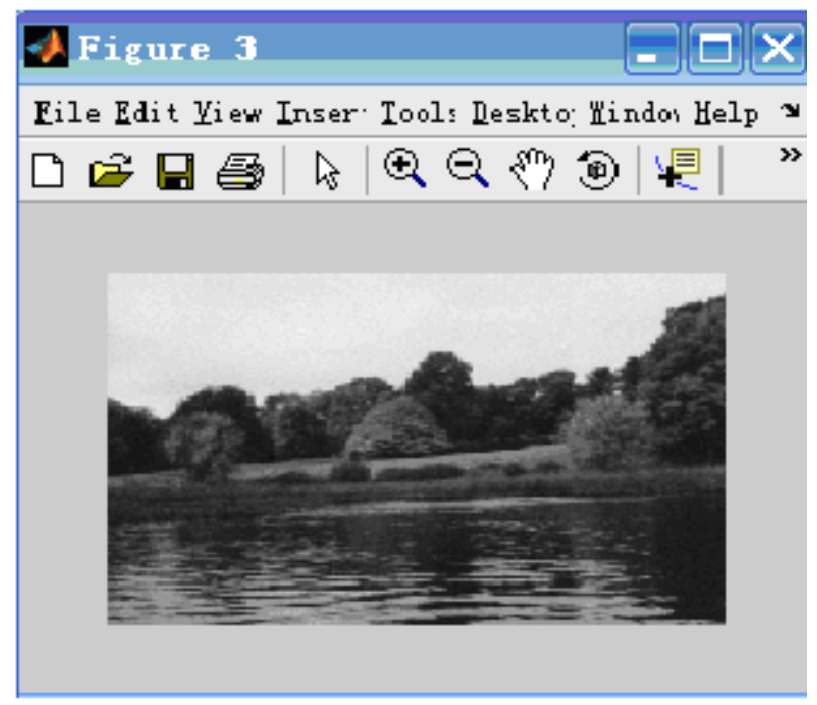

Reconstructed Image

Figure 1. The comparison drugram of compression on DCT 
By comparing the results of image compressions, after the compression of DCT, most of the components have been preserved, so the image can be reconstructed with little distortion.

\section{ACKNOWLEDGMENT}

This work was financially supported by the National Natural Science Foundation of China (61163034, 61373067), the Grassland Excellent Talents Project of Inner Mongolia Autonomous Region ( 2013 ), the supported By Program for Young Talents of Science and Technology in Universities of Inner Mongolia Autonomous Region (NJYT-14-A09), the Inner Mongolia Natural Science Foundation (2013MS0911), the 321 Talents Project the two level of Inner Mongolia Autonomous Region(2010), the Inner Mongolia talent development fund(2011), and the Scientific Research Foundation of Inner Mongolia University For Nationalities (NMDYB15011).

\section{REFERENCES}

[1] HU Qiong,WANG Ronggui,HU Weiwei,YANG Wanting. Color Image Enhancement Based on Histogram Segmentation[J]. Journal of Image and Graphics,2009,14(9):1776-1781.
[2] LI Guanzhang,LUO Wusheng,LI Pei. Color Image Enhancement Based on Visual Characteristics of Human Eyes[J]. OptoElectronic Engineering,2009,36(11):92-95.

[3] Yoon Byoungwoo,Song Woojin.Image Contrast Enhancement Based on the Teneralized Histogram[J].Journal of Electronic Imaging,2007,16(3):033005-033005-8.

[4] GONZALEZ RC,WOODS R E,EDDIN S S L. Digital Image Processing using MATLAB [M].Beijing: Publishing House Of Electronics Industry,2004.

[5] Zhang Yi,Liu Xu,Li Haifeng. Adaptive Image Histogram Equalization Algorithm [J].Journal of ZheJiang University (Engineering Science) .2007, Vol. 41 No.4.

[6] Rafael C. Gonzalez, Richard E. Woods. Digital Image Processing(Third Edition)[M].Ruan Qiuqi, translation. Beijing: Publishing House Of Electronics Industry,2011.6:72-77.

[7] Wang Qiwei. Research on image histogram feature and its application[D].University of Science and Technology of China,2014.

[8] Ruan Qiuqi. Digital Image Processing [M]. Beijing: Publishing House Of Electronics Industry,2001.

[9] Castleman Kenneth R. Digital Image Processing[M].Zhu Zhigang, translation.Beijing: Publishing House Of Electronics Industry, 1998.

[10] Zheng Yongguo. Research and Implementation on image enhancement methods [D]. Shandong University of Science and Technology,2005. 